\begin{tabular}{l|l|l|l|l|l} 
Revista Praxis & ISSN: 1657-4915 & Vol. 13 & No. 1 & 13 - 16 & enero - junio de 2017
\end{tabular}

DOI: http://dx.doi.org/10.21676/23897856.2080

RESEÑA

\title{
SINERGIA DE LA EDUCACIÓN AMBIENTAL Y LA SOCIEDAD EN COLOMBIA
}

\author{
SYNERGY OF ENVIRONMENTAL EDUCATION AND SOCIETY IN COLOMBIA
}

\section{Carlos Alejandro Hernández-Carvajal ${ }^{\text {iD }}$}

$\mathrm{H}_{\mathrm{c}}^{\mathrm{o}}$ oy en día, al hablar de la educación en Colombia, es referirse a una de las problemáticas que más afecta a nuestro país, hablando desde el punto de vista de la calidad (TorrejanoVargas, 2015). Así mismo, esta problemática se ha convertido en un reto para el gobierno nacional, el cual quiere lograr que en 10 años sea la nación más educada de Latinoamérica (Lizarazo, 2017), esto solo se puede alcanzar trabajando mancomunadamente los docentes, estudiantes y padres de familia (García-García, Pulido-Melo, Salazar-Uribe, 2016; Villa \& Villa, 2014), no obstante, el gobierno no debe olvidar la historia de desigualdad del país, la cual se refleja en muchos contextos, incluidos el de la educación, siendo este uno de los problemas arraigados a la baja calidad del sector educativo (Reimers, 2000).

Gálvez (2005) y Cárdenas (2015): afirman que: la calidad es un concepto relativo, cargado de valores, que puede ser variable en cada individuo, dependiendo del tiempo y del espacio. Incluso Di Marco (2015) asegura que: el objetivo fundamental del conocimiento es interpretar la realidad, esto quiere decir que el trabajo en las instituciones educativas debe apuntar hacia la formación de seres dotados de calidad humana, que además de construir conocimientos, puedan tener sentido de responsabilidad como persona educada y preocupada por la sana convivencia.

La educación ambiental, nace a partir de la preocupación por la problemática ambiental en todos sus contextos y por la necesidad de tener una educación distinta que contribuyera en la formación de ciudadanos que sean capaces de construir un futuro sostenible (Meza-Aguilar, 1992),

(...) Sin embargo, a la hora de tomar acciones encaminadas a la prevención y mitigación de impactos ambientales, la comunidad en general no vislumbra el camino a seguir y las normas y leyes que se han planteado para educar a las personas en la preservación del medio ambiente no generan un impacto positivo, debido al desconocimiento de las herramientas de educación ambiental, las cuales permitirían poner en marcha acciones efectivas por parte de la comunidad para el cuidado de su entorno (Pita-Morales, 2016, p.118)

Lo anterior, es un tema preocupante que se ve reflejado en los vacíos de información que existen alrededor de otros tópicos relacionados con las ciencias biológicas, ya que no se puede conservar lo que se desconoce (Vélez, 2013). En este sentido, Colombia posee una gran cantidad de especies, de la cuales no se conocen sus posibles usos o aplicaciones para el manejo de ecosistemas específicos, este es el caso de los hifomicetos acuáticos, que según Pinto \& Smits (2012) "constituyen un grupo heterogéneo de hongos que colonizan, degradan y modifican el material vegetal en el agua, permitiendo que el mismo sea utilizado por otros organismos presentes en el ecosistema acuático, y que en su gran mayoría se desarrollan en aguas de

1. Estudiante del programa de Ingeniería Ambiental y Sanitaria, Facultad de Ingeniería, Universidad del Magdalena, Carrera 32 No. 22 - 08, Santa Marta, Colombia. Correo electrónico: cahdzc26@gmail.com ORCID: 0000-0002-7852-781X 
características óptimas, mientras que otros son tolerantes a condiciones desfavorables, por lo que pueden ser utilizados como indicadores de calidad de agua." Así mismo, "las algas de agua dulce constituyen un grupo diverso de organismos, que aunque suelen tener impactos positivos en los ecosistemas acuáticos, también pueden presentar efectos adversos," puesto que "algunas de ellas producen toxinas que son letales para organismos acuáticos y terrestres (Mogollón, Aycardi, Galeano, Villalobos \& Arango, 2014; Martínez-Silva, 2015).” Por lo tanto es necesario realizar estudios que permitan conocer cuáles son los organismos que generan afectaciones en los diferentes ecosistemas de manera que a futuro se puedan utilizar como indicadores de calidad (Martínez-Silva, 2015) y que la información obtenida a partir de los mismos pueda ser ampliamente divulgada en la comunidad en general.

Caso contrario ocurre con los macroinvertebrados acuáticos, cuyo uso como indicadores de calidad de agua es ampliamente conocido, ya que según Cortés-Guzmán \& Ospina-Torres (2014) "desde un punto de vista funcional, pueden jugar un papel preponderante en la red de interacciones de los ecosistemas acuáticos y en el flujo de recursos alimentarios haciendo que estas comunidades reflejen ciertos cambios en el entorno, permitiendo su uso en estudios de ecología básica y aplicada.”

Teniendo en cuenta lo anterior, cabe mencionar que el desconocimiento de nuestra biodiversidad causa el uso indiscriminado de los recursos naturales y de las especies de flora y fauna que habitan en nuestro país, por esto, es de vital importancia la creación de planes de manejo y medidas para la conservación del medio ambiente que se dicten desde la academia, hacia las comunidades rurales. Estos ejercicios no solo brindan información de primera mano acerca del estado de las poblaciones o las interacciones que las especies pueden tener con el ambiente, sino que también ayudarían a un mejor aprovechamiento de los recursos naturales sobre todo en aquellos espacios donde la explotación es más intensiva (Fabra, Salega, González \& Tavarone, 2014).
Uno de los objetivos principales de la educación ambiental es concientizar a la población en temas sobre el cuidado del ambiente que repercutan positivamente en el ámbito social (Rengifo-Rengifo, Quitiaquez-Segura \& Mora-Córdoba, 2012), como es el caso del uso adecuado de los espacios de esparcimiento y recreación de destino público, tales como: parques, playas, ríos, escenarios deportivos, etc., mediante la aplicación de "programas para incentivar en los usuarios un comportamiento ambientalmente responsable que ayude a aumentar la conciencia ambiental de los visitantes tanto esporádicos como recurrentes" de cada uno de estos lugares, gracias a que este tipo de iniciativas "podrían ser la solución a la presencia de basuras y desechos sólidos" (Noguera-Castro, Botero-Saltarén \& Zielinski, 2012) en la mayoría de los espacios públicos.

Es importante destacar que alternativamente el tema ambiental debe mirarse desde la óptica de los movimientos sociales, explorando los problemas culturales y abordándolos desde los objetivos de la justicia social como lo son: sostenibilidad ambiental e interculturalidad (Núñez, 2012); adicionalmente deben evaluarse las interacciones en el contexto del "paisaje cultural", es decir, las relaciones entre el hombre y su ambiente (Gordillo, 2014; Maita-Agurto \& Minaya-Cabello, 2014).

Otra de las problemáticas que puede ser abordada desde la educación ambiental tiene que ver con el desconocimiento de los riesgos que implica la construcción de edificaciones y todo tipo de infraestructuras a orillas de los ríos, faldas de los cerros, zonas costeras, etc., que son vulnerables a la ocurrencia de desastres producto de los procesos naturales propios de estas zonas tales como desbordamientos, avalanchas, deslizamientos y erosión costera entre otras (Rangel-Buitrago \& Posada-Posada, 2013).

Todo lo anterior, sumado al gran desarrollo de las nuevas tecnologías, le proporcionan a los estudiantes una gran oportunidad para acceder a nuevos conocimientos y crear cambios en su forma de pensar, concebir el mundo, y así lograr trabajar en la consecución de ambientes sanos 
que le proporcione llevar una vida más saludable (Nava, Seijo \& Mier, 2014; Erazo \& Aldana, 2015; Viloria, Bertel \& Daza, 2015; Cardona \& Vaca, 2014).

\section{REFERENCIAS BIBLIOGRÁFICAS}

Cárdenas, J. (2015). La educación sexual como estrategia de inclusión en la formación integral del adolescente. Praxis, 11, 103-115.

Cardona, J., \& Vaca, Y. (2014). Estrategias de la unidad de negocio prestar servicios de formación virtual - Unipanamericana compensar. Praxis, 10, 48-59.

Cortés-Guzmán, D., \& Ospina-Torres, R. (2014). Comunidades de Macroinvertebrados acuáticos en quebradas de la Isla de Providencia, Mar Caribe Colombiano. Revista Intropica, 9, 9-22.

Di Marco, R. (2015). En busca del origen del conocimiento: El dilema de la realidad. Praxis, 11, 150-162.

Erazo, J., \& Aldana, G. (2015). Sistema de creencias sobre las matemáticas en los estudiantes de educación básica. Praxis, 11, 163-169.

Fabra, M., Salega, S., González, C. V., \& Tavarone, A. (2014). "Lo que el agua nos dejó": investigaciones bioarqueológicas en la Costa Sur de la Laguna Mar Chiquita (Córdoba, Argentina). Jangwa Pana, 13, 51-64.

García-García, M. F., Pulido-Melo, C. A., \& Salazar-Uribe, M. O. (2016). Mariposas de otras tierras - El arte como vehículo. Jangwa Pana, 15(1), 105-116.

Gordillo, I. (2014). La noción de paisaje en arqueología. Formas de estudio y aportes al Patrimonio. Jangwa Pana, 13, 195-208.

Lizarazo, T. (2017). Calidad de la educación en Colombia se rajó: 5 puntos sobre 10 . Nueva medición del Ministerio revela que mejores desempeños están en primaria y la media. $E l$ Tiempo. Recuperado de http://www.eltiempo. com/archivo/documento/CMS-15464577

Maita, P. K. \& Minaya, E. E. (2014). El trauma en la piel: El registro de Tatuajes Paracas Necrópolis. Jangwa Pana, 13, 14-33.

Martínez-Silva, P. (2015). Variación espaciotemporal de microalgas acuáticas del embalse de Betania - Huila y su relación con la calidad del agua. Revista Intropica, 10, 11-19.
Meza-Aguilar, L. (1992). Educación ambiental. ¿Para qué? Nueva Sociedad, 122, 176-185.

Mogollón, M., Aycardi, M. P. Galeano, J. Villalobos, J., \& Arango, C. (2014). Variación espacio-temporal de las cianoprocariotas del antiguo delta del río Sinú, Córdoba, Colombia. Revista Intropica, 9, 92-101.

Nava, D., Seijo, C., \& Mier, C. (2014). Inteligencias múltiples: característica congruente del liderazgo ético en entidades financieras, Praxis, 10, 91-99.

Noguera-Castro, L. A., Botero-Saltarén C. M., \& Zielinski, S. (2012). Selección por recurrencia de los parámetros de calidad ambiental y turística de los esquemas de certificación de playas en América latina. Revista Intropica, 7, 59-68.

Núñez, A. (2012). Antropología y patrimonio en el contexto multicultural. Jangwa Pana, $11,40-48$.

Pinto, M., \& Smits, G. (2012). Evaluación preliminar de la riqueza de especies de hifomicetos acuáticos en ríos de la vertiente norte de la costa, Estado Aragua-Venezuela. Revista Intropica, 7, 31-36.

Pita-Morales, L. (2016). Línea de tiempo "Educación Ambiental en Colombia”. Praxis, 12, 118-125.

Rangel-Buitrago, N. G. \& Posada-Posada, B. O. (2013). Determinación de la vulnerabilidad y el riesgo costero mediante la aplicación de herramientas SIG y métodos multicriterio. Revista Intropica, 8, 29-42.

Reimers, F. (2000). Educación, desigualdad y opciones de política en América Latina en el siglo XXI. Revista Latinoamericana de Estudios Educativos, 30(2), 11-42.

Rengifo-Rengifo, B. A., Quitiaquez-Segura, L., \& Mora-Córdoba, F. J. (2012). La educación ambiental una estrategia pedagógica que contribuye a la Solución de la problemática ambiental en Colombia. En Independencias y construcción de estados nacionales: Poder, territorialización y socialización, siglos XIX$X X$. XII Coloquio Internacional de Geocrítica, Bogotá, Colombia.

Torrejano, R. (2015). La educación que merecemos no es la educación que tenemos: el problema de la falta de calidad en la educación básica y secundaria en Colombia 1903-1933. Jangwa Pana, 14, 59-74. 
Vélez, L. (2013). Desconocimiento sobre biodiversidad en Colombia: motor de su deterioro. El colombiano. Recuperado de http://www.elcolombiano.com/historico/ desconocimiento_sobre_biodiversidad_en_colombia_motor_de_su_deterioro-EYEC_265414. Villa, E., \& Villa, W. (2014). Los saberes de la negación y las prácticas de afirmación una vía para la pedagogización desde una perspectiva otra en la escuela. Praxis, 10, 21-36.

Viloria, J., Bertel, M. \& Daza, A. (2015). Percepciones estudiantiles sobre el proceso de acreditación por alta calidad del Programa de Administración de Empresas de la Universidad del Magdalena. Praxis, 11, 89-102. 